\title{
2. Zopiclone, a continuing contribution to the management of sleep disorders
}

\section{MECIIANISM OF ACTION OF ZOPICLONE}

A. Doble 94403 Vitry-sur-Seine, France.

The hypnotic drug zopiclone is a cyclopyrrolone derivative whose mechanism of action involves allosteric modulation of the GABAA receptor Zopiclone displaces the binding of $[3 \mathrm{H}]$-flunitrazepam to the $\mathrm{GABAA}$ receptor with an affinity of $28 \mathrm{nM}$, and enhances the binding of the channel blocker $\left[{ }^{35} \mathrm{~S}\right]$-TBPS. $\left[{ }^{3} \mathrm{H}\right]$-muscimol binding to the receptor is unaffected by zopiclone. Unlike $\beta$-carbolines and zolpidem, zopiclone does not distinguish between $\mathrm{GABA}$ a receptors containing different $\alpha$ subunits $(\mathrm{BZ} 1$ and $\mathrm{BZ2}$ subtypes). Studies with protein-modifying reagents and photoaffinity labelling experiments suggest that zopiclone binds to a domain on the GABAA receptor different from the benzodiazepine binding domain. Photoaffinity labelling of the GABAA receptor with flunitrazepam ( $3 \mathrm{nM}$ for $10 \mathrm{~min}$ ) leads to a near-total loss of benzodiazepine binding, but leaves the binding of zopiclone unaffected. Similarly, inactivation of histidine residues in the receptor with diethylpyrocarbonate $(0.4 \mathrm{mM}$ for $15 \mathrm{~min})$ decrease $\left[{ }^{3} \mathrm{H}\right]$-flunitrazepam binding without affecting cyclopyrrolone binding. Similar data have been obtained upon inactivation of tyrosine residues with tetranitromethane or $\mathrm{N}$-acetylimidazole. The consequence of the interaction of zopiclone with the GABAA receptor is to potentiate responses to GABA. For example, GABA-evoked ${ }^{36} \mathrm{Cl}$ fluxes in rat synaptosomes are enhanced by zopiclone, as are GABA-mediated hyperpolarisations of cultured rat spinal neurones. These effects of zopiclone are reversed by the benzodiazepine antagonist flumazenil. Flumazenil also antagonises the effects of zopiclone in models of anticonvulsant activity and in electroencephalographic studies. Repeated administration of certain benzodiazepines to mice has been shown to produce changes in the "setpoint" of the GABAA receptor, resulting in decreased sensitivity to agonists (tolerance) and increased sensitivity to inverse agonists. It has not been possible to produce such changes in the receptor "set-point" with doses of zopiclone up to $400 \mathrm{mg} / \mathrm{kg}$ i.p
COMPARATIVE EFFECTS OF THREE HYPNOTICS ON PERFORMANCES AND COGNITIVE FUNCTIONS

H. Allain(1), C. Janus (2), P. Raoul (3), A. Beck (3), E. Le Coz (3), A. Pellois (2)

A. Lieury(4), J.M. Gandon (3)

(1) Laboratoire de Pharmacologie, CHRU Pontchaillou - 35033 Rennes Cedex - France (2) Théraplix, Paris - France ; (3) Biotrial S.A., Drug Evaluation and Pharmacology Research, 20, rue du Pr Jean-Pecker, 35000 Rennes - France ; (4) Laboratojre de Psychologie Expérimentale, Rennes - France.

\section{INTRODUCTION}

The research in Clinical Pharmacology must be more and more adapted to European guidelines and recommendations mainly issued from consensus meetings. Hypnotics represent a good example of this question knowing that teams have to publish the feasibility of the theoretically recommended studies and also the possibility to realize the advocated methodological principles. AIM OF THE STUDY

The present study demonstrated the application of the preceding statements while presenting the methodology of a trial in healthy volunteers (HV). The conclusions will insist upon the quality and quantity of the information obtained as well as upon the firm objective of inter-products comparisons useful in therapeutics. DESCRIPTION OF THE STUDY

This double blind placebo controlled study compared the activity of three hypnotics (zopiclone $7.5 \mathrm{mg}$, zolpidem $10 \mathrm{mg}$, flunitrazepam $1 \mathrm{mg}$ ) administered per os as a single dose, on cognitive nocturnal performances in 16 young HV (18-35 y.o.). Pharmacokinetic (PK) parameters were simultaneously measured to establish the relationship between PK and pharmacodynamic (PD) parameters. The design of the study was the following : randomized, latin-square, cross-over, with 4 noctumal assessment periods from 9 p.m. to 8 a.m. (7 days interperiod wash-out). The cognitive tests were two-fold : psychometric tests (CFF), Postural Sway (PS), anterograd memory (Working Memory Task [WMT], Iconic Lexical Treatment [LT], Hemispheric Specialization Task [HST]). Each night, the order was CFF, PS, PK, ILT, HST and WMT, before and at $0 \mathrm{H} 25,0 \mathrm{H} 50,1 \mathrm{H} 25$, $2 \mathrm{H} 05,4 \mathrm{H} 05,7 \mathrm{H} 05$, and $10 \mathrm{H} 05$ post-dosing. CONCLUSION

Such a complex design was performed without any drop-outs over a 3-month period of time. The psychometric tests could be done even in this night procedure. Interdrug comparisons could be made. The relationship between PK and PD have been computerized authorizing modelization and so prospective statements. 
EFFECTS OF ZOPICLONE ON PSYCHOMOTOR AND INFORMATION PROCESSING SKILLS INYOLYED IN SKILLED PERFORMANCE J. F. O'Hanlon

Institute for Human Psychopharmacology, University of Limburg, Maastricht, The Netherlands

Before and after its introduction in 1987, zopiclone has been the object of investigation in more than 15 psychometric studies employing both young and elderly healthy volunteers and insomniac patients. Their primary purpose was in all cases the same, to determine whether nocturnal doses (usually the standard $7.5 \mathrm{mg}$ ) possess residual sedative effects capable of interfering with skilled, safety-relevant performance, such as car driving, over the following day. In general, the results have shown little or no residual effects. When some significant effect was found its magnitude was always below a level that could be considered as clinically relevant and never did it extend more than 12 hours from the time of dosing. The review describes zopiclone's generally benign residual effects on skilled performance and goes on to indicate what information is still lacking.
A METHODOLOGICAL APPROACH OF HYPNOTICS WITHDRAWAL SYMPTOMATOLOGY IN HUMANS : AN IULUSTRATION WITH ZOPICLONE P. Lemoine (1). H. Allain (2), J.M. Gandon, A. Pellois. C. Janus

(1) C.H. LE VINATIER, 95 Bd Pinel , 69677 BRON Cedex (France)

(2) CHRU de PONTCHALLLOU, Laboratoire de Pharmacologie, Av. du Pr Léon Bernard, 35043 RENNES Cedex (France)

Prospective and good quality clinical trials comparatively assessing hypnotics withdrawal have to systematically cope with difficulties which can only be solved by complex studies. Some unpublished trials in this field help us to elaborate rational experimental design.

The first problem concerns the admitted treament duration of insomnia by a hypnotic, knowing that French legislation does not recommend more than 4 week long periods of treatment; on the other hand, until now, chronic insomnia treatment implied 6 months at least. This requirement comes from the supposed correlation between treatment duration versus frequency and severity of withdrawal signs. A second difficulty (linked to the preceding) has to deal with recruitment and randomization of the subjects. The chronic treatment period is a therapeutical phase, necessarily and by definition an open one. The analysis of the withdrawal period, for a mere compound is a unique trial by itself. If a comparison is made with another hypnotic, a real second trial, simultaneously carried out, is therefore performed. As a consequence, and according to the classical assessment criteria for withdrawal (description of the events, carly dropouts, Ashton symptoms list, Tyrer questionnaire, sleep assessments ...) the number of included patients is obviously high (for example, 400 for comparing zopiclone and zolpidem). Another difficulty is linked to the fragility of chronic insomniac patients and the fact that the mere inclusion in a trial immediately induces phenomena frequently and wrongly considered as withdrawal symptoms ("trial effect"). In a unpublished trial, we observed that during a single-blind nun-in period wih the same drug, a high frequency of drop-outs occured. That means that a run-in period is necessary or that one has to weight the events occuning during the first days after inclusion. The high quality of events notification has to be stressed during this initial phase of the trial

These data imply that withdrawal symptomatology assessments need large scale studies and careful interpretations.
ZOPICLONE AS A CATALYST TO HYPNOTIC WITHDRAWAL
C. Shapiro
Department of Psychiatry, University of Toronto, The Toronto Hospital 399 Bathurst Street, Toronto, Ontario MST 258, Canada.

Withdrawal from hypnotics can produce a variety of problems, especially sleep difficulties, some of which may arise from the multiple actions of most hypnotics, thus producing a range of rebound effects. This paper examines whether switching patients to a hypnotic with a narrower range of action and of a different class would reduce these problems.

One hundred and thirty-four patients participated in the first aspect of these studies, they were randomly allocated to one of three methods of switching from "previous hypnotic" to zopiclone (a cyclopyrrolone). The methods were GAP (an interval between taking the two drugs); ABUTTAL (taking zopiclone immediately on stopping previous drugs); and OVERLAP (gradually reducing previous drug after starting zopiclone). The main findings were that zopiclone was associated with better sleep and increased alertness; the abuttal method was the easiest method of switching and no serious side effects on zopiclone were reported. It was concluded that zopiclone has a useful role in benzodiazepine withdrawal and that immediate substitution is better tolerated, the chance of success may depend on the age of the patient, the nature and duration of the sleep problems and the type and dose of benzodiazepine being replaced.

In a subgroup of the former study $(n=9)$ sleep recordings were carried out at the time of presentation, at the end of a month on zopiclone and at the end of a month after hypnotic withdrawal. The results show that in certain features of sleep e.g. REM latency, there is a normalisation of the pattern during zopiclone which persists to the time of the third study, i.e. after the patient is off all hypnotics. For other features of sleep architecture e.g. sleep onset there are dramatic gains while on zopiclone but there are lost on withdrawing the zopiclone. Finally certain features e.g. slow wave sleep percentage dramatically increase with the switch to zopiclone and are partially but significantly retained in the withdrawal period.

In evaluating the long term effectiveness of the process of using zopiclone as a catalyst for hypnotic withdrawal, ninety-nine of the 134 patients completing the switch over the study were interviewed 14-21 months after the initial switch to zopiclone. Eighty-one had remained off hypnotic drugs. These results indicated a better success rate for hypnotic withdrawal than other studies in the literature.
ZOPICLONE IN THE TREATMENT OF INSOMNIA IN DEPRESSED PATIENTS

Dr. M. Van Moffaert Department of Psychiatry and Centre for Sleep and Wake Disorders, University Hospital, De Pintelaan 185, 9000 Gent, Belgium

Sleep problems and depression are intertwined. Rather than an epiphephomenon of affective illness sleep disturbances are an essential part of its pathogenesis. An overview of clinical observational data, polysomnographic studies and the antidepressant effect of sleep deprivation, indicate the intimate relationship between sleep disturbances and depression. During depression the polysomnogram shows specific REM changes with a total sleep advance, correlating with parallel circadian shifts of other physiological phenomena e.g. a shift of body temperature. Monitoring the effect of the tricyclic antidepressant on REM with polysomnography will contribute to the prediction of the eventual treatment response. Total sleep deprivation, partial slecp deprivation and phase advance manipulation bring about temporary changes in the depressive mood. Most clinically effective antidepressant drugs inhibit REM sleep. Zopiclone is an attractive hypnotic that may be administered for short term treatment of sleep disturbances in depressed patients treated with a recently started antidepressant. 
SLEEP QUALTTY AND DAYTIME WELL-BEING IN SLEEP-DISTURBED PATIENTS : A COMPARATIVE STUDY IN 1570 PATIENTS

G. Hajak, E. Rujther

Psychiatric Clinie, University of Gottingen, 37075 Gottingen, Germany

In a randomized, double-blind, parallel group study in private practice, zopiclone given for 28 days was compared with flunitrazepam, triazolam and placebo on quality of sleep and daytime well-being in patients with insomnia. The responder rate tended to be higher with zopiclone (37.4\%) than with flunitrazepam (30\%) and triazolam $(32.2 \%)$ and was significantly greater $(p=0.0017)$ than with placebo $(26.8 \%)$

Daytime well-being was particularly responsive to zopiclone and most responsive in severe insomniacs. With the exception of those to triazolam, rates of response were most pronounced in patients with insomnia of a short duration (less than one year) than in those with insomnia of a longer duration (more than one year) Following discontinuation of treatment, all treatment groups showed only a moderate reduction in therapeutic effect, but no rebound insomnia occurred. However, after treatment discontinuation 20-40 percent of patients had values below baseline in some response criteria, this was also observed in the placebo group.
EFFECTS OF ZOPICLONE ON SLFEP-EEG AND DAYTIME WELL-BEING IN THE ELDERLY

J. Kummer $-\frac{1}{2}$ L. Gìndel 2 , B. Seyffer $\stackrel{2}{2}$

1 Gerontopsychiatrische Klinik, 16225 Eberswaide

${ }^{2}$ Landesnervenklinik Nordschwarzwald, 75365 Calw, Germany

Sleep disorders and the associated affection of daytime well-being are encountered more frequently in elderly patients compared to other population groups. In most cases benzodiazepines (BZD) are prescribed, even though they often affect the sleep stages already impaired in elderly patients. Therefore, new hypnotics should also be expected to prevent these negative effects in elderly patients with sleep disorders. Here the first non-benzodiazepine hypnotic, zopiclone, may constitute a useful alternative approach.

A double-blind, randomized sleep-EEG study of zopiclone $(Z)$ vs. placebo (P) with special cmphasis on daytime well-being, was performed. The study involved 14 inpatients (mean age : 73 years, no dementia nor depression) with sleep disorders. After a 4-day placebo washout phase, the patients were given $Z$ (7.5 $\mathrm{mg}$ ) (8 patients) or $\mathrm{P}$ ( 1 capsule) ( 6 patients) for 21 days before going to bed followed by 4 days of placebo administration to evaluate withdrawal effects. To assess efficacy, sleep EEGs were performed on the days $-2,-1,1,10,21,23$ and 25. Daytime well-being was assessed every two days during the treatment phase by the Quatember-Maly's test.

Based on the EEG, it was shown that $Z$ reduces the awake periods and prolongs the TST as compared with $P$. Regarding the various sleep stages, $Z$ led to reduction in sleep onset latency and a prolongation of sleep stage II without any influence being noted on REM sleep. After discontinuing $Z$, merely the awake period was prolonged the night after, but reduced again in the second night. No marked rebound signs occurred. Zopiclone seems to be a useful alternative to the BZD in managing sleep disorders in the elderly. 